SELECTED PAPER AT NCSP'15

\title{
Ball Trajectory Extraction in Team Sports Videos by Focusing on Ball Holder Candidates for a Play Search and 3D Virtual Display System
}

\author{
Junji Kurano, Masaki Hayashi, Taiki Yamamoto, Hirokatsu Kataoka, \\ Masamoto Tanabiki, Junko Furuyama and Yoshimitsu Aoki \\ Graduate School of Science and Technology, Keio University \\ 3-14-1 Hiyoshi, Kohoku-ku, Yokohama 223-8522, Japan \\ Phone/FAX: +81-45-566-1454 \\ E-mail: \{jkurano, mhayashi, tyamamoto, kataoka\}@aoki-medialab.org, \\ \{tanabiki.masamoto, furuyama.junko\}@jp.panasonic.com, aoki@elec.keio.ac.jp
}

\begin{abstract}
When team sports coaches instruct their team members, a sample video is needed for an offensive instruction. However, it is very difficult to search a video for the parts of interest, and it takes a long time to compile a database, for example, ball trajectories and player locations, because sports videos have many periods with no play such as timeouts. In particular, information on the ball trajectory is very important for tactical analysis. However, it is currently inputted manually, which is time-consuming. Therefore, we focus on American football, where the ball can rarely be seen. The contribution of this paper is the submission of a method of automatic ball trajectory extraction. First, we remove the no-play periods in team sports videos to leave only play time and reduce the processing time. Second, we extract the ball trajectory to enable tactical analysis. We propose a new approach to ball tracking by focusing on the ball holder prediction. Our method can be applied to situations with heavy occlusion. Finally, we construct a play search and 3D virtual display system using information on the ball trajectory and the Unity, which is the development environment of the 3D virtual visualization. We used it to construct a Virtual 3D map for watching the play from an arbitrary viewpoint.
\end{abstract}

\section{Introduction}

Recently, many applications for a sports video analysis have been proposed. Such as for automatic tactical analysis [1], and formation analysis [2]. For these applications, player locations and the ball trajectory are required in team sports videos. In particular, information on the ball trajectory is very important for match analysis. However, while a player's location can be obtained automatically in sports videos such as soccer and ice hockey [3], the ball trajectory is generally obtained manually. In American football and rugby videos, it is extremely difficult to extract the ball trajectory using a method of player/ball tracking [3][4] because the ball is held by players and can rarely be seen in the video, resulting in heavy occlusion. Therefore, novel methods for extracting play time and automatic ball tracking are needed.

\section{Related Work}

Ball trajectory extraction [5] has already been applied to many sports. For example, in tennis, information on the ball trajectory is used to judge whether a serve was successful or not. However, in tennis, the appearance of the ball is very clear, and there is seldom heavy occlusion by players. Therefore, ball trajectory extraction in tennis is easier than in team sports, such as soccer, and American football. In addition, many previous studies have attempted to track the ball's location using only the result of ball detection, as shown in [6]. In [6], information from an RGB histogram and the ball's shape are used to construct a likelihood function, which suggests a method of tracking the ball using a particle filter. However, it is very difficult to track the ball using only its color and shape information because of occlusion.

Kim et al. extracted the global motion tendency reflecting a scene by tracking the movements of objects in a video of an football game [2]. This method can detect a player's general actions in a sports video.

Wang proposed a method of tracking the ball using information about the ball holder [4]. They used multiple cameras and assigned a player as the ball holder using a conditional random field (CRF), which consists of ball 


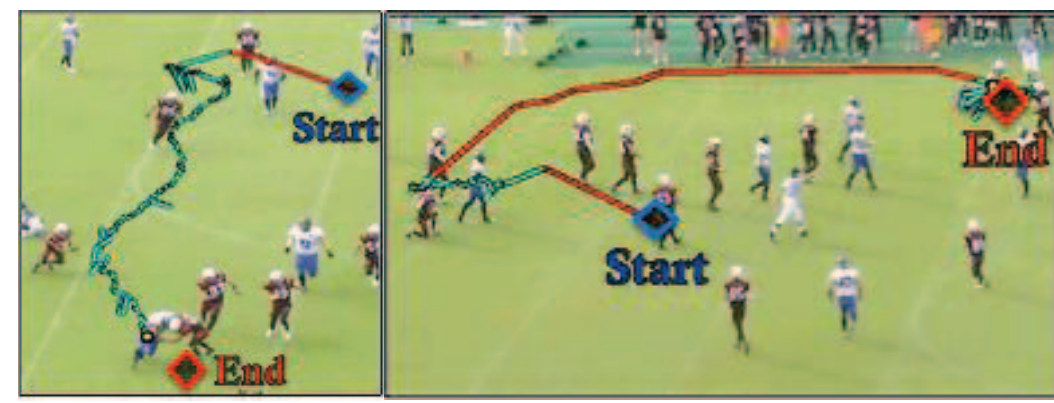

Figure. 1: Ball trajectory in team sports

(red line: ball trajectory, blue line: ball holder trajectory)

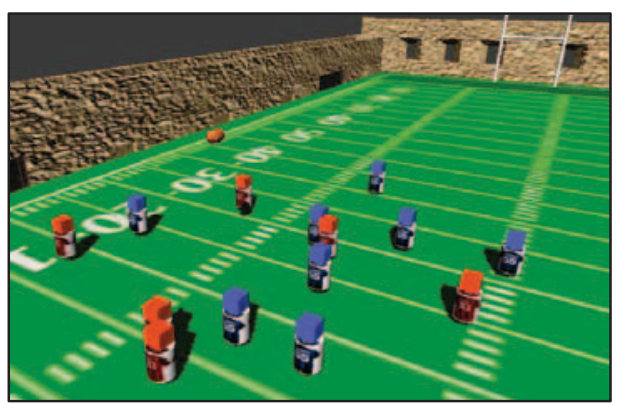

Figure. 2: The result of play search shown in the form of virtual 3D map detection and player tracking. However, this work relies considerably on the ball detection result, making it difficult to apply to many sports, for example, American football, in which the ball is often occluded by many players.

\section{Proposed Method}

To reduce the time required for analyzing matches, we perform play-time detection. After that, to predict the ball holder, we calculate the play start/end points and detect all the players. In addition, we detect the ball when it can be clearly seen. Moreover, we predict the ball holder using information obtained from our likelihood function, and we combine the results of ball detection and ball holder tracking. Finally, for intuitive understanding, we construct a play search system using the ball trajectory and a 3D map using the Unity, which is the development environment of the $3 \mathrm{D}$ virtual visualization.

\subsection{Play-time detection}

In team sports videos, especially those of American football, the play time can be separated. This means that the start/end times of the play can be detected. In American football videos, the flow of the play consists of four components:

1) Some of the players arrange themselves in the initial formation, which is called the line of scrimmage. Then, most of the players stand still. 2) The ball is thrown from the center of the line of scrimmage, and then all the players start moving together. 3) The play lasts until the ball leaves the field or the ball holder is pushed down.

We use this characteristic of the play to extract the foreground region by subtracting the pixel value between frames. Then, we detect the time that the values of the foreground region increase sharply and consider this as the start of play time. In addition, we calculate the velocity of all players using the scalar of the dense optical flow [7]. Then, we detect end of the time using information that its differentiation doesn't change so much, and consider this as the end of the play time. The thresholds are set by considering results of a preliminary experiment.

\subsection{Detecting players/ball and calculating start/end points}

We construct a detector for players using a histograms of oriented gradients (HOG) [8] and AdaBoost [9], and a condition for ball detection using information on the color, size, and shape of the ball as shown Eqs. (1)-(3).

$$
\left(L_{e}=1\right) \cap\left(\left(\frac{1}{2} L_{c}+\frac{1}{2} L_{s}\right) \geq 0.75\right)
$$

$L_{s}$ : Likelihood of the size (labeling)

$L_{e}$ : Oval fitting result ( $L_{e}=1$ means true)

$L_{c}$ : Likelihood of the color (color histogram matching)

$$
L_{c}=1-\sqrt{1-\frac{1}{\sqrt{\overline{H_{1}} \overline{H_{2}} N^{2}}} \sum_{I} \sqrt{H_{1}(I) \cdot H_{2}(I)}}
$$

$N$ : Bin number of the color histogram

$H_{1}, H_{2}$ : Registered/compared color histograms

$$
L_{s}=\exp \left(-\frac{(S-225)^{2}}{2}\right)
$$

\section{$S$ : Size of the labeling region}

Furthermore, we calculate the play start/end points. First, to calculate the start point of play, we construct a detector for the initial formation using the HOG and the AdaBoost. We use the center of the detection region as the start point of play. Second, to calculate the end point of play, we use two heat maps of the players' density and direction concentration. The way in which play ends in team sports varies, meaning that a universal method of end point detection or machine learning cannot be applied easily. 
Therefore, we divide the field into $200 \times 200$ regions (not pixels) called the field grid, and calculate the players' density $L_{\text {density }}$ given by Eq. (4) using the result of player detection.

$$
\begin{aligned}
& L_{\text {density }}=\frac{1}{L_{\text {densitv Max }}} \cdot \frac{R_{p}}{R} \\
& R_{p}: \text { Size of the players' region }
\end{aligned}
$$

Moreover, we use the dense optical flow to calculate the point towards which the players are converging. In our method, the results of the dense optical flow are divided into eight directions, and we calculate the direction concentration $L_{\text {direction. }}$ using Eqs. (5)-(7).

$$
\begin{gathered}
\left.L_{\text {direction_direct }}\right|_{1 \leq x \leq 200,1 \leq y \leq 200}=\sum \frac{1}{\text { dis }_{\text {grid }}} \\
L_{\text {direction }} \text { opposite }\left.\right|_{1 \leq x \leq 200,1 \leq y \leq 200} \\
=\sum-d i s_{\text {grid }}+\text { flowsize } \\
\left.L_{\text {direction }}=\frac{1}{L_{\text {direction }_{\text {Max }}}\left(L_{\text {direction }}\right. \text { direct }}+L_{\text {direction_opposite }}\right)
\end{gathered}
$$

$x, y$ : Field grid coordinates

dis grid: Distance from each field grid

flowsize: Number of field grids

Finally, the results for the players' density $L_{\text {density }}$ and the direction concentration $L_{\text {direction }}$ are used to calculate the likelihood of the end point of the play $L_{\text {terminal }}$ given by Eq. (8), and the point with the highest likelihood is considered as the point at which play ends.

$$
L_{\text {terminal }}=\left.\frac{1}{3} t_{\text {last }} \cdot L_{\text {density }}\right|_{t=t_{\text {last }}}+\sum_{t=\frac{2}{3} t_{\text {last }}}^{t_{\text {last }}} L_{\text {direction }}
$$

$t_{\text {last }}:$ Frame when play ended

\subsection{Ball holder estimation}

To avoid errors in ball holder prediction, we choose the ball holder candidates using the results of ball detection and the calculation of the start point of play. After that, we predict the ball holder. To predict the ball holder, we use three likelihoods: 1) the likelihood of the approach receive, 2 ) the likelihood of team identification, 3) the likelihood of the distance from the end point of play.

\subsubsection{Likelihood of the approach receive}

In team sports, the ball holder is always the focus of attention, so the direction of all players converge to the ball holder. Therefore, we use the heat map of the direction concentration to calculate the likelihood of the approach receive using Eq. (9).

$L_{\text {approach }}=\frac{\sum_{y=p y \_m i n}^{p y \_m a x} \sum_{x=p x \_m i n}^{p x \_m a x} L_{\text {direction }}(x, y)}{L_{\text {approach_Max }}}$

$L_{\text {direction }}:$ Likelihood of approach receive

$L_{\text {direction_Max }}$ : Maximum likelihood of approach receive px,py_min/max : Coordinate of directed player

\subsubsection{Likelihood of team identification}

Each attacking/defending team player wears the same uniform, so they can be clearly identified. Therefore, the players in a team sports video are identified using a color histogram, and we define a likelihood of the team identification $L_{\text {team }}$ using Eq. (10). The team variable $R$ is defined as attacking team $A$, and defending team $B$.

$$
L_{\text {team }}=\frac{1}{L_{\text {team_Max }}} \cdot\left(1-\left(\frac{H_{R=B}}{H_{R=A}}\right)\right)
$$

$H$ : Result of color histogram

3.3.3 Likelihood of the distance from the end point of play

When the play in a team sport ends, ball holder estimation is especially difficult because we use information on the distance from the end point of the play, and we define the likelihood as $L_{\text {terminal_distance, }}$ given as follows.

$$
\begin{aligned}
& L_{\text {terminal_distance }} \\
& \qquad=\frac{1}{L_{\text {terminal_distance_Max }}} \cdot \frac{1}{\text { dis terminal }}
\end{aligned}
$$

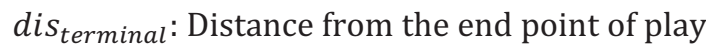

Finally, to predict the ball holder, we use a likelihood function including the three above likelihoods, and the player with the highest ball holder likelihood $L_{\text {holder }}$ is considered as the ball holder given Eq. (12)-(13). When the ball holder likelihood function is calculated, we use a weighting function.

$$
\begin{aligned}
& L_{\text {holder }} \\
& =a_{1}(t) L_{\text {team }}+a_{2}(t) L_{\text {terminal_distance }}+a_{3}(t) L_{\text {approach }} \\
& \left(0 \leq L_{\text {holder }}, L_{\text {team }}, L_{\text {terminal_distance }}, L_{\text {approach }} \leq 1\right)(12) \\
& a_{1}(t)=-\frac{0.2}{t_{\text {last }}} t+0.2, a_{2}(t)=\frac{0.4}{\left(0.7 t_{\text {last }}\right)^{2}} t^{2} \\
& a_{3}(t)=1-a_{1}(t)-a_{2}(t) \\
& (\text { pass }=\varphi) \|(\text { pass } \neq \varphi \cap \text { after pass }) \\
& a_{1}(t)=-\frac{0.2}{t_{\text {last }}} t+0.2, a_{2}(t)=0, \\
& a_{3}(t)=1-a_{1}(t)-a_{2}(t)(\text { pass } \neq \varphi \cap \text { before pass })
\end{aligned}
$$




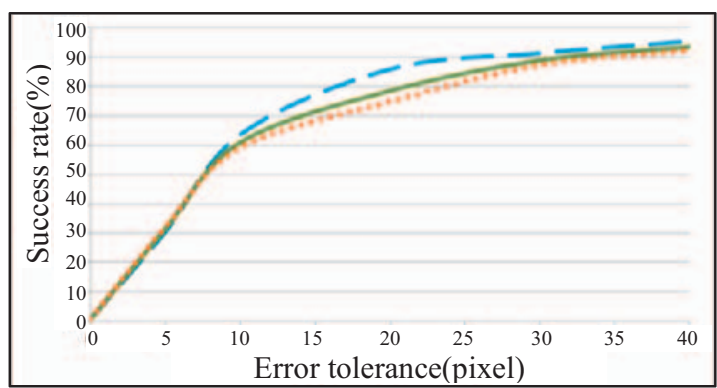

Figure. 3: Accuracy of our method (solid line: average of all play, dashed line: average of play with passing, dotted line: average of play without passing)

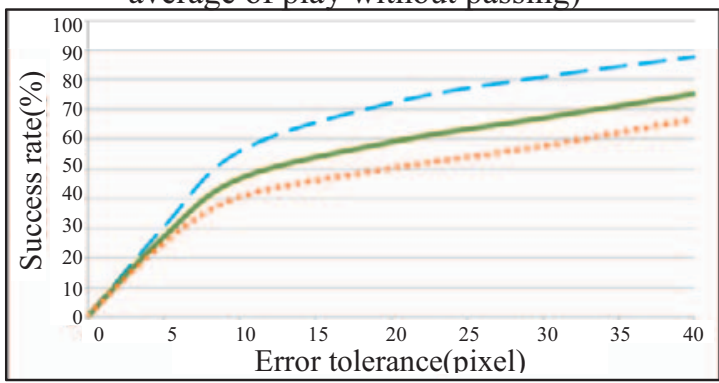

Figure. 4: Accuracy of the method using a particle filter (solid line: average of all play, dashed line: average of play with passing, dotted line: average of plav without passing)

\subsection{Construction of play search system and 3D virtual display system}

We constructed a play search system using the information on the ball trajectory. Our system requires four points on a bird's-view of the field of play as an input, and each point is compared with ball trajectory database using the Euclidean distance. Furthermore, we construct a 3D virtual display system for watching sports videos more effectively. The height of the ball was inputted manually.

\section{Experiments}

We performed three experiments. First, we compared our result with the ground truth. Second, we compared ours with the tracking method of a particle filter [10], where the model parameter is linear motion. Third, we compared with a previous method of extracting the ball trajectory [11]. We used the same 10 videos in each experiment and calculated the Euclidean distance from the ground truth in each frame. The results are shown in Figs. 3-5.

\section{Conclusions}

This paper describes the extraction of the ball trajectory in team sports videos where heavy occlusion occurs such

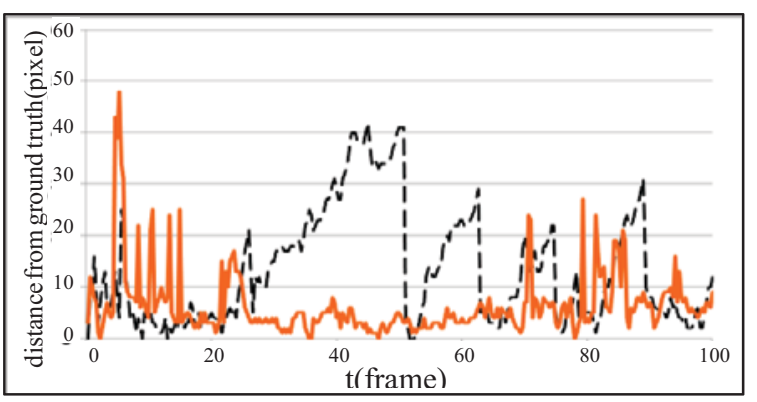

Figure. 5: Comparative experiment result

(solid line: our method, dashed line: method [111) as in American football. Moreover, we constructed a play search/3D virtual display system using information on the ball trajectory. In the future, we will combine our method with a method of player number recognition to improve the tracking precision.

\section{Acknowledgment}

All videos used in this paper are of matches of the American football team Panasonic Impulse. We would like to thank Panasonic for providing the videos.

\section{References}

[1] H. Ito, T. Takiguchi and Y. Ariki: Player tracking on monocular video using time situation graph, MIRU, OS13-03, 2012.

[2] K. Kim, D. Lee and I. Essa: Detecting regions of interestin dynamic scenes with camera motions, CVPR, pp. 1258-1265, 2012.

[3] TRACAB (http://www.tracab.com/technology.asp).

[4] X. Wang, V. Ablavsky, H. B. Shitrit and P. Fua: Take your eyes off the ball: Improving ball-tracking by focusing on team play, CVIU, 2013.

[5] Hawk-Eye (http://www.hawkeyeinnovations.co.uk/).

[6] T. Nishino, T. Takiguchi and Y. Ariki: Situation recognition using $3 \mathrm{D}$ positional information of ball from monocular soccer image sequence, IRU, 2009.

[7] G. Farneback: Two frame motion estimation based on polynomial expansion, SCIA, 2003.

[8] N. Dalal and B. Trigs: Histograms of oriented gradients for human detection, CVPR, 2005.

[9] P. Viola and M. Jones: Rapid object detection using a boosted cascade of simple features, CVPR, 2001.

[10] M. Isard and A. Blake: Condensation - conditional density propagation for visual tracking, Int. J. Comput. Vis, Vol. 29, No. 1, pp.5-28, 1998.

[11] J. Kurano, T.Yamamoto, H. Kataoka, M. Hayashi and Y. Aoki: Ball tracking in team sports by focusing on ball holder candidates, IWAIT, 2014. 